\title{
The Burden of Influenza Virus Infection among Children under 12 Years Old in Tropical Countries
}

\author{
Lim Kai Joo ${ }^{1,2}$, Mohd Yusof Ibrahim²* Mohd Rohaizat Hassan³ ${ }^{3}$ Eric Tan Chee How², Mohammad Saffree Jeffree ${ }^{2}$
}

${ }^{1}$ Sabah Women and Children's Hospital,

Ministry of Health, Sabah, Malaysia

2 Department of Public Health Medicine,

Faculty of Medicine and Health Sciences,

Universiti Malaysia Sabah, Kota Kinabalu,

Sabah, Malaysia

${ }^{3}$ Department of Community Health,

Faculty of Medicine,

Universiti Kebangsaan Malaysia,

Cheras, Kuala Lumpur, Malaysia

*Corresponding author's email:

dr.myusof@ums.edu.my

Received: 19 March 2021

Accepted: 20 August 2021

Keywords: influenza, paediatric, children, neurology complication, breastfeeding, seasonality

\section{ABSTRACT}

Every year influenza epidemic causes about 3 - 5 million severe respiratory infections and $\mathbf{2 5 0 , 0 0 0}$ to 500,000 deaths worldwide. Among them about 28,000 - 111,500 (11 - 22\%) children under 5 years old die because of influenzarelated acute lower respiratory tract infections. This review aims to explore the burden, risk factors and seasonality related to influenza virus infection among children under 5 years old in tropical countries. From 1st to 31 st December 2020 literature search was done on PubMed and ScienceDirect databases using 'influenza' and other keywords such as paediatric, children, neurology complication and breastfeeding for articles published in the English language. About $10 \%$ of hospitalization with respiratory infections in children $<18$ years old are related to influenza. It is reported that about $11 \%-26 \%$ of febrile illnesses among outpatients and 6 $-14 \%$ of hospitalized patients with pneumonia were due to influenza virus. Global paediatric mortality estimates that death caused by influenza is highest among 1 - 12 months old children which is about $\mathbf{2 . 8 \%}$ of all deaths. In Malaysia, there are no seasonal variations of influenza and the prevalence is higher during the middle of the year. Risk factors include male, age <12 months old and presented with seizure. Breastfeeding and vaccination for pregnant mothers can be beneficial for infants up to 6 months old. Influenza among the paediatric group is a significant health burden even in healthy children and are likely to be underdiagnosed. Vaccination can also be focused on pregnant mothers and children less than 12 years old. 


\section{INTRODUCTION}

Among respiratory infections influenza virus infection causes severe disease for those less than 5 years old and is one of the major contributors of morbidity and mortality (Zhou et al., 2012). Influenza disease is one of the main causes of admission in paediatric groups globally and it is a vaccine-preventable disease. Recognition of severe influenza especially in the paediatric group in the developing country had been increased due to an increase in laboratory capacity (Nair et al., 2011). Influenza is infectious and caused by influenza viruses that infect the respiratory system and potentially become pandemic. Children with underlying medical illnesses are particularly at risk for influenza infection and related complications such as pneumonia. Influenza A and $B$ viruses frequently affected children with an attack rate of about $18 \%$ in children younger than 3 years old (Alsuwaidi et al., 2017). Children also contribute to the influenza disease transmission in the community due to their prolonged viral shedding compared to adults. In one study it was found that neuraminidase inhibitors (NAls) can significantly shorten the duration of fever in children infected with the influenza virus even though virus shedding is still detected after defervescence (Kondo et al., 2016). Hence, these patients might still be infectious and could spread the infection even the fever subsided (Kondo et al., 2016).

Most of the articles on paediatric influenza from developed countries with temperate climates has shown defined seasonal peak, especially in winter. However, there are fewer studies done in tropical countries due to the perception that influenza infection is not a serious health problem and the lack of diagnostic tools. This perception changed when the H1N1 pandemic occurred in 2009. In Malaysia, the H1N1 pandemic in 2009 raised awareness and concern among the public as well as healthcare staff that influenza infection is an important disease. Hence, this article reviews the burden of influenza among children, associated risk factors and seasonality of influenza in tropical countries including Malaysia.

In this article, we reviewed published papers on influenza infection among children. We searched articles from PubMed and ScienceDirect database from 1 st to $31 \mathrm{st}$ December 2020 with keywords of 'influenza', 'paediatric', 'children', 'neurology complication' and 'breastfeeding'. The search field was restricted to title only to produce a manageable number of papers. Only English papers were included and studies on animals were excluded. We further searched for publications relevant to our focus on paediatric groups especially in tropical countries including Malaysia.

\section{The Burden of Influenza in Children}

Worldwide, influenza was associated with $10 \%$ (95\% Cl 8 - 11\%) of admission with respiratory diseases in children less than 18 years old, whereby $5 \%$ were from children less than 6 months old and about $16 \%$ were among children $5-17$ years old (Kathryn et al., 2016). Every year influenza epidemics cause 3 to 5 million severe illnesses and about 290,000 to 650,000 deaths (WHO, 2011). There were an estimated 28,000 - 111,500 deaths in children younger than 5 years old contributed by influenza-related acute lower respiratory tract infections (ALRI) in 2008, most of this occurred in developing countries (Nair et al., 2011). It is estimated that about 90 million (95\% Cl 49 - 162 million) occurred in children older than 1 year old and about 20 million (13 - 32 million) episodes of influenzaassociated ALRI occurred in children aged 0 - 4 years (Nair et al., 2011). From this, there is an estimated 1 million new episodes of influenzaassociated severe disease occurred in children younger than 5 years in the year 2008 (Nair et al., 2011). In Thailand, across all age groups between 2005 and 2008 have shown that influenza virus infection was associated with $10.4 \%$ of hospitalised pneumonia cases and 
the highest incidence was seen mainly in children under 5 years old and adults over 75 years old (Sam, 2015; Simmerman et al., 2009). Hospitalization rates for children below 5 years old with seasonal influenza were estimated to be 1.4 per 100,000 in the USA (Bourgeois et al., 2009), countries in Southeast Asia rates vary in different countries such as Singapore (0.7 - 0.9) (Koh et al., 2016), Thailand (2.4) and Vietnam (3.9 - 4.7) (Sam, 2015). The incidence of influenza-associated ALRI among children less than 6 months was roughly 1.6 times higher than children of $6-11$ months. There are an estimated $228,000(95 \% \mathrm{Cl} 150,000$ to 344,000 ) influenza-associated hospitalizations per year among children aged less than 6 months old (Kathryn et al., 2016).

In Malaysia, the H1N1 pandemic in 2009 has alerted the public and healthcare staff that influenza is an important infectious disease and should not be taken lightly (Sam, 2015). The first detection of pandemic influenza was reported in May 2009 in Malaysia (Balraj et al., 2011). Most of the cases occurred during the 6 weeks from August to September 2009 whereby there were 12,307 reported cases with 77 deaths in 2009 (Sam, 2015). A total of 1,362 children aged $<12$ years old were hospitalized for influenza-like illness (ILI) from 18 June 2009 until 1 March 2010. Among 1,362 children, 134 (9.8\%) required intensive care and 51 (3.7\%) died whereby 25 (49\%) were less than 2 years old. Severe disease leading to death was more likely seen in patients who had at least one concurrent condition such as chronic lung disease, neuromuscular disease, cardiovascular disease, renal disease, immunosuppression, obesity and malnutrition (Muhammad Ismail, 2011). The age group less than 2 years old had a hospitalization rate of 33 per 100,000 children (0.1 pre-pandemic) and a mortality rate of 1.3 per 100,000 children (0.1 pre-pandemic) (Sam, 2015).

Studies on the burden of influenza virus infection in Southeast and East Asia are limited, diagnosis by PCR has improved the detection of influenza virus infection among cases with respiratory illness (James et al., 2008). Studies from different countries have reported that $11-26 \%$ of outpatient with febrile illness and $6-14 \%$ of hospitalized pneumonia cases had laboratory-confirmed influenza virus infection (Simmerman \& Uyeki, 2008). Influenza illness is a burden among children below 5 years old in which these findings are consistent with many papers and school-aged children (5- 17 years old) contributed a higher proportion of influenza-associated hospitalization (Kathryn et al., 2016). Global paediatric mortality estimates that $2.8 \%$ of all deaths are in those aged $1-12$ months which is the highest proportion even though this estimation can be underestimated because the analysis of severe influenza infection presented with non-respiratory clinical presentation such as seizure is not captured (Lozano et al., 2012).

A recent study done in China reported coinfection of SARS-CoV-2 and influenza viruses in patients during the COVID-19 outbreak. This study found that $42.7 \%(131 / 307)$ of total patients had a single infection of the SARS-CoV-2 virus and the remaining had coinfection with either influenza A (49.8\%) and $B(7.5 \%)$ viruses. This increased not only the burden of influenza but also the burden in combating the pandemic especially the co-infection caused poorer prognosis and higher mortality in the vulnerable group. Besides that, patients coinfected with SARCoV-2 and influenza $B$ virus seem to have a higher risk of developing poor outcomes and presented with more severe symptoms such as fatigue, abnormalities on chest computed tomography (CT) or decreased lymphocytes (Yue et al., 2020).

\section{Seasonality}

Generally, influenza virus infection occurred throughout the year in tropical countries such as Malaysia with no clear seasonality (Sam, 2015). This is supported by Koh and colleagues whereby seasonality is less clear for influenza 
virus compared to the respiratory syncytial virus (RSV) which presented with an annual epidemic with a strong seasonal pattern (Koh et al., 2016). In 2005 - 2009, influenza viruses were commonly isolated from May to August (Saat et al., 2010). Influenza A is more commonly detected compared to influenza B. Influenza $A$ was seen throughout the year with peak activity in May while influenza B virus activities were seen more in between November and March (Khor et al., 2012). In Malaysia, the southern hemisphere vaccine is recommended but there is limited data to show the matching of influenza strain and vaccine. For example, a study done by Saat et al. (2010) showed in between 2005 and 2009, the trivalent vaccine only matched 2 out of 5 years (the year 2006 and 2008) with the predominant circulating strain whereas vaccine for the years 2005, 2007 and 2009 were not compatible. However, for the years 2005 and 2006, influenza A (H1N1) strains circulating in Malaysia were compatible with the strain used in the WHO southern hemisphere vaccine (Saat et al., 2010).

The incidence of seasonal influenza remains unknown in tropical countries. Many studies reported that influenza viruses in tropical countries such as Malaysia were found to be all year-round with higher occurrence in the middle of the year. Another study also mentioned that influenza cases reported no seasonality with peaking between November - January and May - July (Koh et al., 2016). There are usually few influenza virus strains (3 to 6) co-circulating simultaneously every year. Influenza vaccine components are based on antigenic and genetic analysis of recent influenza virus isolates worldwide. Thus, surveillance activities are important to ensure a good match between vaccine strains and circulating strains of the influenza virus (Saat et al., 2010).

Comparing to our neighbouring country such as Thailand, influenza A with pneumonia hospitalizations increased especially during June to October in 2005, 2006 and 2008.
However, this pattern was not observed in 2007 whereby influenza A activity increased from January to April 2007. In 2008, influenza $A$ and B both occurred throughout 2008. Influenza B virus infections did not present with seasonal patterns. In the same paper, they suggested that a routine influenza vaccination policy be implemented to tackle post-hospital discharge specific groups as they noticed that there is an underestimation of influenza pneumonia incidence. Successful detection of influenza virus with high-quality specimens will help to improve reporting of influenza infection (Simmerman et al., 2009).

\section{Risk Factors and Influenza Virus Infection Among Children}

Influenza virus infection increased with age from $<6$ months old to $1-5$ years old which is significantly infected in older children (Sam et al., 2010). This is different from the respiratory syncytial virus (RSV) whereby it is the commonest identified respiratory virus in young children particularly $<6$ months old but declined in those aged $1-5$ years old (Bourgeois et al., 2009; Khor et al., 2012). Another study showed that severe influenza was associated with age < 12-monthold whereby $25.6 \%$ of this age group had severe influenza. Although influenza usually occurred in older children, age $<12$ months old children with influenza have a higher risk of severe disease. Besides that, absences of rhinitis at presentation and female sex can be associated with severe influenza and should not rule out influenza infection (Sam et al., 2010). There is also another report that showed that absence of nasal congestion is associated with admission thinking of those with nasal congestion can be upper respiratory tract infection compared to young infant presented with fever, but no other focus infection will be hospitalized presumed bacterial sepsis. The most commonly infected ethnic groups were Malays (61.5\%), Chinese (20.6\%) and Indians (16\%) which reflected in sampled ethnicity (Koh et al., 2016). Another paper showed that 
male gender is associated with hospitalization (Quach et al., 2003); similar to a few other papers showed that male was higher among positive influenza cases (Khor et al., 2012); Lin et al., 2012(Koh et al., 2016). Non-modifiable such as gender and ethnicity contribute to the occurrence of influenza infection among children. The Male (39.9\%) gender had higher positive influenza compare to females (32.1\%) (Lin et al., 2012). Sam and Aizuri et al. (2010) showed that $56.8 \%$ of severe influenza diseases in hospitalized children were male (I. Sam et al., 2010). This was also observed in another study by Koh et al. (2016) whereby male children have contributed $60 \%$ positive for pandemic 2009 influenza. However, this finding contradicted with WHO study whereby females were 1.6 times less likely to survive H5N1 infection compared to males (Klein et al., 2012).

A study done in Thailand showed that the incidence of influenza pneumonia was highest in less than 5 years old. About $8 \%$ of pneumonia cases in children less than 5 years old and $18.9 \%$ cases in children $5-9$ years of age were influenza-positive (Simmerman et al., 2009). This is consistent with others study whereby influenza infection occurrence increased with the age of children and tend to happen in older children (Khor et al., 2012). This is because children of school-going age were more active with activities at school and highly contacting with other children. The same study also mentioned that children less than 6 years old showed significant vaccine efficacy compare to older children because younger children have a higher vaccination coverage rate (Suzuki et al., 2014). Aged 3 - 5 years old formed the largest group to be infected with the influenza virus during the influenza pandemic 2009. In their study also mentioned that $85.7 \%$ of the children were given antiviral oseltamivir (Koh et al., 2016). Studies showed that children treated with Neuraminidase inhibitors (NAls) such as peramivir, oseltamivir, zanamivir and laninamivir have a shorter duration of fever than untreated patients (Dobson et al., 2015;
Saito et al., 2010). Hence, those patients who received treatment had return to school or work earlier than untreated patients (Kondo et al., 2016). The pandemic of 2009 has caused severe illnesses such as Acute Respiratory Distress Syndrome (ARDS) and encephalitis whereby about $10 \%$ of the children needed intensive care (Koh et al., 2016). Influenza-related neurologic complications occurred in $10 \%$ of the children hospitalized with confirmed influenza infection. Seizure is the commonest presented symptom observed among influenza-related neurologic complications. The mechanism is unknown whether the influenza virus caused seizure directly or by fever associated with the infection (Newland et al., 2006). However, Influenza $A$ is one of the causes of febrile fit (Chiu et al., 2001). Among patients hospitalized with laboratory-confirmed influenza infection, about $1 \%$ had influenza encephalopathy however none of them died. The finding was in contrast with Japan's study whereby there was a $25 \%$ to $37 \%$ mortality rate in Japan (Newland et al., 2006; Togashi et al., 2004). During the H1N1 pandemic, severe illness was seen in both healthy and those children with underlying illness (Koh et al., 2016). Children with seasonal influenza infection showed a longer hospitalization duration (Bagdure et al., 2010). Koh and colleagues also mentioned that those children who have been infected with the influenza virus should have been vaccinated for Streptococcus pneumonia and Hemophilus influenza because these bacterial infections can increase mortality (Koh et al., 2016).

Another study done in Taiwan showed that factors such as family members infected with influenza, fever duration $>38^{\circ} \mathrm{C}$, age of children $>5$ years old, myalgia, lethargy, sore throat, cough, and headache were reviewed significantly associated with positive influenza cases. The same study presented that age more than 10 years old had a higher risk of positive influenza whereby this infection is easily spread in school and may have cluster infection. In addition, adolescents are also able to describe their influenza-like symptoms 
compare to younger children and adolescents do not seek medical attention if only have mild discomfort (Lin et al., 2012). Besides, older aged children are also easier to obtain highquality specimens compare to young children.

Breast milk protects against many respiratory tract infections including influenza. Influenza virus infection will not spread to infants through breast milk. The transmission of influenza is by respiratory droplets especially when people cough, sneeze, talk, or touch on the contaminated surface. A mother's breast milk is unique which contains antibodies that can protect her infant to get the flu. Breast milk is rich in nutrition and a solely nutrient for a baby less than 6 months old especially for those exclusive breastfeeding mothers. The study also showed that influenza vaccination to give above 6 months old because of the passive maternal antibody. The same study also showed that mothers who received influenza vaccine had the highest antibodies during delivery and remained significant amount in serum breast milk up to 6 months (Schlaudecker et al., 2013). Hence, the pregnant mother is encouraged to get an influenza vaccine. Influenza vaccine is safe for breastfeeding mothers. Women who get the flu vaccine during pregnancy or breastfeeding will develop antibodies against flu that can be shared with infants through breast milk. If the mother is too ill to give breastfeeding, she can express the breast milk and give the infant through a bottle. Breast milkis the best source of nutrition for infants. Infants who are breastfed are less vulnerable to getting infections including severe respiratory illnesses than infants who are not breastfed. When a mother has flu, her breastmilk contains antibodies that can help to protect her infant from flu. Annual flu vaccination is recommended for those aged 6 months and older whereas for children younger than 6 months, the person around the infant including caregivers should receive the flu vaccination. For women who are breastfeeding with suspected influenza infection can be treated with Oseltamivir as data indicate that Oseltamivir is excreted in breast milk with a minimal amount (DeTora \& Malkowski, 2019).

\section{CONCLUSION}

Influenza infection among the paediatric group is a significant health burden even in healthy children and are likely to be underappreciated. Influenza activities were seen year-round in Malaysia with biannual peaks. Influenza vaccine may benefit pregnant mothers and infants up to 6 months old if widely used as routine vaccination during antenatal follow up. Influenza vaccination also should be focused on Malaysian children below 5 years old especially when intranasal influenza vaccines are available.

\section{CONFLICT OF INTEREST}

All authors declare there is no conflict of interest in preparing this article.

\section{FUNDING}

This study was funded in part by a UMS Great Research Grant (GUG0383-1/2019) from Universiti Malaysia Sabah.

\section{ACKNOWLEDGEMENTS}

We would like to thank the Director General of Health Malaysia for the permission to publish this article and gratefully acknowledge the staff of Sabah State Health Department, Sabah Women and Children's Hospital, Universiti Malaysia Sabah and Universiti Kebangsaan Malaysia for their support and contribution in this article.

\section{REFERENCES}

Alsuwaidi, A. R., Al-Mekaini, L. A., Kamal, S. M., Narchi, H., \& Souid, A. K. (2017). Seroprevalence of influenza $A$ and $B$ viruses among unvaccinated children in the United Arab Emirates: A cross-sectional study. BMC Research Notes, 10 (379), 1 - 4. https://doi. org/10.1186/s13104-017-2720-8 
Bagdure, D., Curtis, D. J., Dobyns, E., Glodé, M. P., \& Dominguez, S. R. (2010). Hospitalized children with 2009 pandemic influenza A (H1N1): Comparison to seasonal influenza and risk factors for admission to the ICU. PLOS ONE, 5 (12). https://doi.org/10.1371/ journal.pone.0015173

Balraj, P., Sidek, H., Suppiah, J., Khoo, A. S. B., \& Saat, Z. (2011). Molecular analysis of 2009 pandemic Influenza A (H1N1) in Malaysia associated with mild and severe infections. Malaysian Journal of Pathology, 33 (1), 7 - 12.

Bourgeois, F. T., Valim, C., Mc Adam, A. J., \& Mandl, K. D. (2009). Relative impact of influenza and respiratory syncytial virus in young children. Pediatrics, 124 (6), e1072 - e1080. https://doi. org/10.1542/peds.2008-3074

Chiu, S. S., Tse, C. Y., Lau, Y. L., \& Peiris, M. (2001). Influenza $A$ infection is an important cause of febrile seizures. Pediatrics, 108 (4). https:// doi.org/10.1542/peds.108.4.e63

DeTora, L. M., \& Malkowski, J. A. (2019). Pregnant? You need a flu shot! Women's Health Advocacy, 111 - 121. https://doi. org/10.4324/9780429201165-14

Jason, G. N. V., Matt, L., Anna, W. R., Kateri, H., Daniel, J. L., Ron, K., Theoklis, E. Z., Barbara, W., Richard, L. H., \& Susan, E. C. (2006). Neurologic complications in children hospitalized with influenza: Characteristics, incidence and risk factors. The Journal of Pediatrics, 150 (3), 306 - 310. https://doi. org/10.1016/j.jpeds.2006.11.054

Kathryn, E. L., Harish, N., Mohammad Hafiz, R., Fatima, V., Robert, B., Mahmudur, R., Paul, K., Hongjie, Y., Guiselle, G., Daouda, C., Julio, A., Daddi, J., Stephen, R. C. H., William, A., Ricardo, M., \& Mandeep, C. E. (2016). Global role and burden of influenza in pediatric respiratory hospitalizations, 1982 - 2012: A systematic analysis. PLOS Medicine. 13 (3), 1 19. Doi: 10.1371/journal.pmed.1001977

Khor, C. S., Sam, I. C., Hooi, P. S., Quek, K. F., \& Chan, Y. F. (2012). Epidemiology and seasonality of respiratory viral infections in hospitalized children in Kuala Lumpur, Malaysia: A retrospective study of 27 years. BMC Pediatrics, 12. https://doi.org/10.1186/14712431-12-32

Klein, S. L., Hodgson, A., \& Robinson, D. P. (2012). Mechanisms of sex disparities in influenza pathogenesis. Journal of Leukocyte Biology. https://doi.org/10.1189/jlb.0811427
Koh, M. T., Eg, K. P., \& Loh, S. S. (2016). Hospitalised Malaysian children with pandemic ( H1N1) 2009 influenza: clinical characteristics, risk factors for severe disease and comparison with the 2002 - 2007 seasonal influenza. Singapore Medical Journal, 57 (2), 81 - 86. https://doi.org/10.11622/smedj.2015146

Kondo, H., Shobugawa, Y., Hibino, A., Yagami, R., Dapat, C., Okazaki, M., \& Saito, R. (2016). Influenza virus shedding in laninamivirtreated children upon returning to school. Tohoku Journal of Experimental Medicine, 238 (2), 113 - 121. https://doi.org/10.1620/ tjem.238.113

Lin, C., Chi, H., Lin, H., Chang, L., Hou, J., Huang, C., \& Chiu, N. (2012). Original article a scoring system for predicting results of influenza rapid test in children: A possible model facing overwhelming pandemic infection. Journal of Microbiology, Immunology and Infection, 45 (4), 271 - 275. https://doi. org/10.1016/j.jmii.2011.11.016

Lozano, R., Naghavi, M., Foreman, K., Lim, S., Shibuya, K., Aboyans, V., \& Memish, Z. A. (2012). Global and regional mortality from 235 causes of death for 20 age groups in 1990 and 2010: a systematic analysis for the Global Burden of Disease Study 2010. Lancet (London, England), 380 (9859), 2095 - 2128. https:// doi.org/10.1016/S0140-6736(12)61728-0

Muhammad Ismail, H. I. (2011). Characteristics of Children Hospitalized for Pandemic (H1N1) 2009, Malaysia. Emerging Infectious Diseases, 17 (4), 2010 - 2012. https://doi.org/10.3201/ eid1704101212

Nair, H., Brooks, W. A., Katz, M., Roca, A., Berkley, J. A., Madhi, S. A., \& Campbell, H. (2011). Global burden of respiratory infections due to seasonal influenza in young children : A systematic review and meta-analysis. The Lancet, 378 (9807), 1917 - 1930. https://doi. org/10.1016/S0140-6736(11)61051-9

Quach, C., Piché-Walker, L., Platt, R., \& Moore, D. (2003). Risk factors associated with severe influenza infections in childhood: Implication for vaccine strategy. Pediatrics, 112 (3 Pt 1). https://doi.org/10.1542/peds.112.3.e197

Saat, Z., Abdul Rashid, T. R. T., Yusof, M. A., Kassim, F. M., Thayan, R., Kuen, L. S., \& Saraswathy, T. S. (2010). Seasonal influenza virus strains circulating in Malaysia from 2005 to 2009. Southeast Asian Journal of Tropical Medicine and Public Health, 41 (6), 1368 - 1373. 
Saito, R., Sato, I., Suzuki, Y., Baranovich, T., Matsuda, R., Ishitani, N., \& Suzuki, H. (2010). Reduced effectiveness of oseltamivir in children infected with oseltamivir-resistant influenza A (H1N1) viruses with His275Tyr mutation. Pediatric Infectious Disease Journal, 29 (10), 898 - 904. https://doi.org/10.1097/ INF.0b013e3181de9d24

Sam, I., Abdul-murad, A., Karunakaran, R., Rampal, S., Chan, Y., Marie, A., \& Ariffin, H. (2010). Clinical features of Malaysian children hospitalized with community-acquired seasonal influenza International Journal of Infectious Diseases Clinical features of Malaysian children hospitalized with community-acquired seasonal influenza. International Journal of Infectious Diseases, 14 (May 2014), e36 - e40. https://doi. org/10.1016/j.ijid.2009.10.005

Sam, J. I. (2015). The burden of human influenza in Malaysia. Med J Malaysia, 70 (3), 127 - 130. PMID: 26248773.

Schlaudecker, E. P., Steinhoff, M. C., Omer, S. B., McNeal, M. M., Roy, E., Arifeen, S. E., \& Zaman, K. (2013). IgA and neutralizing antibodies to influenza A virus in human milk: A randomized trial of antenatal influenza immunization. PLoS ONE, 8 (8), 6 - 13. https:// doi.org/10.1371/journal.pone.0070867

Simmerman, J. M., \& Uyeki, T. M. (2008). The burden of influenza in East and South-East Asia: A review of the English language literature. Influenza and Other Respiratory Viruses, 2 (3), 81 - 92. https://doi.org/10.1111/j.17502659.2008.00045.x
Simmerman, J. M., Chittaganpitch, M., Levy, J., Chantra, S., Maloney, S., Uyeki, T., \& Chunsuttiwat, S. (2009). Incidence, seasonality and mortality associated with influenza pneumonia in Thailand: 2005 2008. PLoS ONE, 4 (11), 2005 - 2008. https:// doi.org/10.1371/journal.pone.0007776

Suzuki, T., Ono, Y., Maeda, H., Tsujimoto, Y., Shobugawa, Y., Dapat, C., \& Saito, R. (2014). Effectiveness of trivalent influenza vaccine among children in two consecutive seasons in a community in Japan. Tohoku Journal of Experimental Medicine, 232 (2), 97 - 104. https://doi.org/10.1620/tjem.232.97

Togashi, T., Matsuzono, Y., Narita, M., \& Morishima, T. (2004). Influenza-associated acute encephalopathy in Japanese children in 1994 - 2002. Virus Research, 103 (1 - 2), 75 - 78. https://doi.org/10.1016/j. virusres.2004.02.016

WHO. (2011). Seasonal influenza and influenza A (H1N1). Retrieved from http://www.who.int/ ith/diseases/si_iAh1n1/en/

Yue, H., Zhang, M., Xing, L., Wang, K., Rao, X., Liu, H., \& Shang, J. (2020). The epidemiology and clinical characteristics of co-infection of SARS-CoV-2 and influenza viruses in patients during COVID-19 outbreak. Journal of Medical Virology, 92 (11), 2870 - 2873. https://doi.org/10.1002/jmv.26163

Zhou, H., Thompson, W. W., Viboud, C. G., Ringholz, C. M., Cheng, P. Y., Steiner, C., \& Shay, D. K. (2012). Hospitalizations associated with influenza and respiratory syncytial virus in the United States, 1993 - 2008. Clinical Infectious Diseases, 54 (10), 1427 - 1436. https://doi.org/10.1093/cid/cis211 